\title{
Tratamiento periodontal regenerativo en dientes con afectación furcal
}

\section{Regenerative periodontal procedure in teeth with furcation involvement}

\author{
FABRIZI S* \\ ORTIZ-VIGÓN CARNICERO A* \\ BASCONES-MARTÍNEZ A**
}

\author{
Fabrizi S, Ortiz-Vigón Carnicero A, Bascones-Martínez A. Tratamien- \\ to periodontal regenerativo en dientes con afectación furcal. Av \\ Periodon Implantol. 2010; 22, 3: 147-156.
}

\section{RESUMEN}

Debido a las condicionantes anatómicas y a su relativa inaccesibilidad para el control de placa por parte del paciente los dientes con afectación furcal representan un reto para los clínicos. Con el fin de tratar estas áreas de difícil manejo, se ha estudiado un amplio rango de modalidades de tratamiento entre las cuales destacan: el desbridamiento mecánico quirúrgico y no quirúrgico, la plastia furcal, la tunelización, la hemisección, la resección radicular y los tratamientos regenerativos, sin resultados concluyentes.

Objetivos: revisar la evidencia disponible sobre la naturaleza de la regeneración periodontal obtenible en molares con afectación furcal y evaluar el efecto de la regeneración sobre los parámetros clínicos periodontales.

Conclusión: la regeneración periodontal en lesiones furcales mediante RTG y/o EMD son técnicas efectivas y predecibles para la mejoría clínica y pronóstico de los molares con lesiones de furcación.

PALABRAS CLAVE: Regeneración en furcas, lesiones furcales.

\section{SUIMIMARY}

Because of anatomical conditioners and their relative inaccessibility for plaque control by patients, teeth with furcal affectation represents a challenge for clinicians. With the purpose of dealing with these difficult handling areas, an ample rank of treatment modalities have been studied between which can be emphasized: surgical and nonsurgical mechanical debridement, furcal plastia, tunelization, tooth hemi-section, radicular resection and regenerative treatments without obtaining conclusive results.

Objectives: to review the available evidence on the nature of achievable periodontal regeneration in molars with furcal affectation and to evaluate the effect of regeneration through periodontal clinical parameters.

Conclusion: periodontal regeneration in furcal lesions by means of RTG and/or EMD are effective and predictable technique for the clinical and prognostic improvement of molars with furcación lesions.

KEY WORDS: Regeneration in furcas, furcales injuries.

Fecha de recepción: 30 de junio de 2009.

Aceptado para publicación: 4 de julio de 2009.

* Odontólogo. Alumno Máster de Periodoncia. Facultad de Odontología. Universidad Complutense de Madrid.

** Catedrático de Medicina Bucal y Periodoncia. Departamento de Medicina y Cirugía Bucofacial. Facultad de Odontología. Universidad Complutense de Madrid. España. 


\section{INTRODUCCIÓN}

La etiología primaria de la enfermedad periodontal es la presencia y la maduración del biofilm dentario. $\mathrm{El}$ tratamiento de la periodontitis está enfocado en detener el desarrollo de dichas condiciones mediante la terapia mecánica subgingival, estableciendo unos niveles locales bacterianos compatibles con la salud gingival (Socransky y Haffajee, 2005). Algunos estudios han mostrado que la periodontitis crónica puede ser tratada con éxito mediante terapia quirúrgica y no quirúrgica. El mantenimiento a largo plazo de estos resultados favorables es condición sine qua non para un adecuado control de placa establecido durante la terapia periodontal de mantenimiento (Axelsson, Nystrom y cols., 2004). Debido a las condicionantes anatómicas y a su relativa inaccesibilidad para el profesional y para el control de placa por parte del paciente los dientes multirradiculares representan un reto para los clínicos
(Lang, Cumming y cols., 1973; Fleischer, Mellonig y cols., 1989). En pacientes susceptibles de periodontitis la pérdida de inserción puede progresar hasta el punto de la exposición de la región furcal. Estudios sobre cadáveres humanos refieren que la prevalencia de dientes molares con afectación varía del $26-30 \%$ (Bissada and Abdelmalek, 1973) mientras estudios longitudinales aportan datos ligeramente mayores, siendo del $35 \%$ en molares mandibulares y $90 \%$ en molares maxilares (Ross and Thompson, 1980). "Una furca se define como un área anatómica de un diente multirradicular donde las raíces divergen" y una lesión furcal se refiere a "la reabsorción patológica del hueso en esta área" (AAP 1992).

Se han descrito múltiples clasificaciones de las lesiones furcales basadas en el grado de sondaje horizontal y vertical. Las clasificaciones más comúnmente empleadas son las que aparecen en la tabla l. Con el objetivo

\section{TABLA 1.- CLASIFICACIONES DE LAS LESIONES DE FURCA}

\begin{tabular}{|l|l|}
\hline \multirow{2}{*}{ Glickman (1953) } & $\begin{array}{l}\text { Grado I. Bolsa en la entrada de la furca pero, hueso furcal intacto. } \\
\text { Grado II. Pérdida de hueso interradicular sin extension al lado opuesto. } \\
\text { Grado III. Pérdida ósea de lado a lado. } \\
\text { Grado IV. Pérdida ósea de lado a lado sin tejidos blando interpuestos. }\end{array}$ \\
\hline Goldman (1958) & $\begin{array}{l}\text { Grado I. Incipiente. } \\
\text { Grado II. Cul-de-sac (fondo de saco) } \\
\text { Grado III. De lado a lado. }\end{array}$ \\
\hline \multirow{3}{*}{ Hamp y cols. (1975) } & $\begin{array}{l}\text { Grado I. Pérdida de soporte periodontal Horizontal menor a } 3 \text { mm. } \\
\text { Grado II. Pérdida de soporte horizontal }>\text { a } 3 \text { mm pero sin traspasar. } \\
\text { Grado III. Pérdida horizontal de lado a lado. }\end{array}$ \\
\hline Ramfjord \& Ash (1979) & $\begin{array}{l}\text { Clase I. Incial. Destruction <2 mm (<1/3 de la anchura) } \\
\text { Clase II. Cul-de-sac. }>2 \text { mm (>1/3 de la anchura) sin traspasar } \\
\text { Clase III. De lado a lado. }\end{array}$ \\
\hline Tarnow \& Fletcher (1984) & $\begin{array}{l}\text { Subclasificación basada el grado de pérdida vertical } \\
\text { Subclase A. 0-3 mm } \\
\text { Subclase B. 4-6 mm } \\
\text { Subclase C. }>7 \text { mm }\end{array}$ \\
\hline Fskow and Kapin (1984) & $\begin{array}{l}\text { Mismas subclases que Tarnow \& Fletcher (1984), por tercios } \\
\text { Combina la clasificación de Glickman y Hamp igual del I al IV pero subdivide. }\end{array}$ \\
\hline Ricchetti (1982) & $\begin{array}{l}\text { El grado II en l (<3 mm) y 2 (>3 mm) } \\
\text { Clase I. l mm de pérdida de soporte. } \\
\text { Clase Ia. l-2 mm de pérdida horizontal. } \\
\text { Clase II. 2-4 mm de pérdida horizontal. } \\
\text { Clase IIa. 4-6 mm de pérdida horizontal. } \\
\text { Clase III. } 6 \text { mm de pérdida horizontal. }\end{array}$ \\
\hline
\end{tabular}


de tratar estas áreas de difícil manejo, se han estudiado un amplio rango de modalidades de tratamiento entre las cuales destacan: el desbridamiento mecánico quirúrgico y no quirúrgico, la plastia furcal, la tunelización, la hemisección, la resección radicular y los tratamientos regenerativos. Según una revisión recientemente publicada (Huynh-Ba, Kuonen y cols., 2009), que analiza estudios con un mínimo de cinco años de seguimiento, la tasa de supervivencia a largo plazo de molares con afectación furcal varía según el tipo tratamiento.

En las lesiones de furcas de grado I (Fig. 1) el raspado y alisado radicular muestra buenos resultados mantenibles a largo plazo. En los estudios donde se analiza el tratamiento quirúrgico sin afectación dental se observa una gran variabilidad de los resultados con respecto a la tasa de supervivencia resultante de cada uno de ellos.

Los valores más bajos son los del estudio de McFall y cols. en 1982 con un $43 \%$ y los mayores con un $96 \%$ (Svardstrom and Wennstrom, 2000). Dentro de los estudios que analizan la tunelización, como opción de tratamiento, destacan los estudios de Hamp y cols., 1975 con un $57,1 \%$, el estudio de Little y cols., 1995, con un 89\% y el estudio de Dannewitz y cols., con un $92,9 \%$.

En relación a los estudios en los que se analiza la cirugía periodontal resectiva (resección radicular y/u odontosección) las tasas de supervivencia van de un $57,9 \%$ (Dannewitz, Krieger y cols., 2006) hasta un 98,4\% (Carnevale, Pontoriero y cols., 1998). En la mayoría de estos estudios se evidencia que la principal causa de pérdida dentaria posterapéutica son las caries, los fracasos endodónticos y las fracturas radiculares. Por úl-

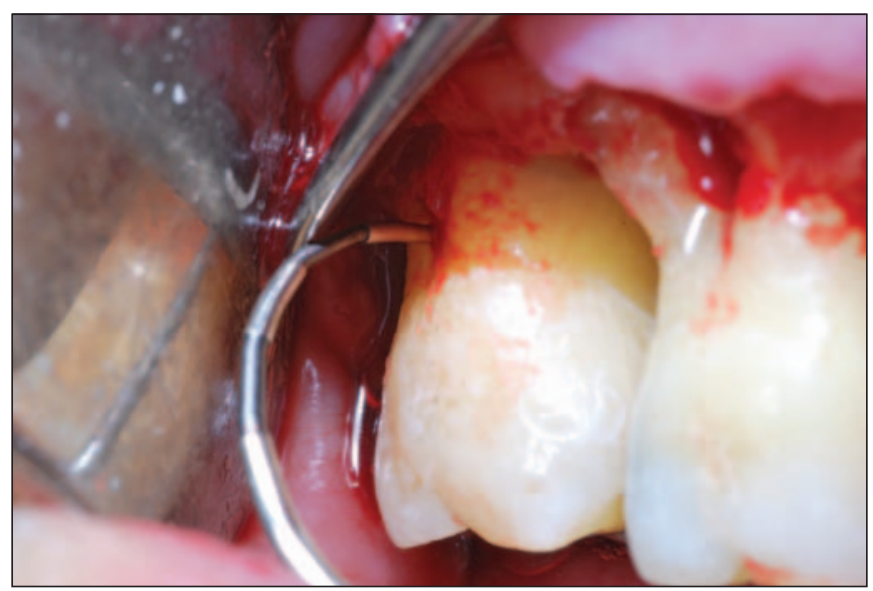

Fig. 1: Lesión de furca de grado I. timo evaluando la tasa de supervivencia en estudios con un enfoque regenerativo.

El estudio de Yukna y Yukna (1997) aborda el tratamiento regenerativo, de furcaciones de grado II mandibulares y maxilares, mediante un colgajo desplazado coronalmente junto con un injerto óseo, obteniendo tras un periodo de 6 años un 100\% de supervivencia. En otros estudios el tratamiento de elección es la regeneración tisular guiada (RTG). Los resultados obtenidos demuestran una tasa de supervivencia de un $100 \%$ a 5 años (Eickholz and Hausmann 2002), de un 98,1\%, a 5 años en el de Dannewitz y cols. en 2006 y del 83,3\% a 10 años en el de Eickholz y cols., 2006. Pese a la relevancia de los resultados encontrados en los estudios de regeneración en molares con afectación furcal, hay que considerar que en estos se utiliza como variable respuesta principal la tasa de supervivencia a medio-largo plazo. Esta variable no aporta datos precisos en cuanto al tipo de cicatrización, característica de la inserción, grado de regeneración y estado clínico del diente tratado. "La terapia periodontal regenerativa tiene como objetivo, restaurar el aparato de soporte del diente que ha sido perdido por trauma o por enfermedad periodontal"' (Sculean, Nikolidakis y cols., 2008).

Definiendo regeneración periodontal verdadera a aquel procedimiento o técnica que conlleve a la formación de nuevo LP, nuevo cemento acelular y nuevo hueso con fibras conectivas insertadas evaluados histológicamente. El abordaje quirúrgico convencional y el raspado y alisado radicular acaban cicatrizando con un epitelio largo de unión (Caton and Greenstein, 1993). Actualmente los procedimientos que han sido capaces de demostrar una regeneración periodontal verdadera son los injertos óseos autógenos, la regeneración tisular guiada (Nyman, Lindhe y cols., 1982), y las proteínas de la matriz del esmalte (EMD). El principal objetivo de cualquier tipo de terapia sobre las lesiones de furcación es obtener el cierre completo de las mismas; si esto no resulta posible se procuraría obtener como resultado posterapéutico una lesión más superficial (Sanz and Giovannoli, 2000) dirigiendo nuestros objetivos terapéuticos a obtener un relleno óseo parcial del defecto, ganancia del nivel de inserción clínico y reducción de la profundidad de sondaje.

\section{OBJETIVOS}

El objetivo de este artículo es revisar la evidencia disponible sobre la naturaleza de la regeneración periodontal obtenible en molares con afectación furcal y 
evaluar el efecto de la regeneración sobre los parámetros clínicos periodontales clásicos intentando contestar a las siguientes cuestiones:

1. ¿Existe evidencia de regeneración en modelos preclínicos?

2. ¿Existe evidencia de regeneración en modelos clínicos?

3. ¿Existe predictibilidad en cuanto a la regeneración de lesiones furcales de distinto grado: I, II y III maxilares y mandibulares?

\section{¿EXISTE EVIDENCIA DE REGENERACIÓN EN MODELOS PRECLÍNICOS?}

La cicatrización de los defectos furcales tras el tratamiento de cirugía de acceso junto con injertos óseos ha sido estudiado en modelos animales (Ellegaard, Karring y cols., 1973; Ellegaard, Karring y cols., 1975). En estos estudios, realizados en monos, se utilizaron varios tipos de injertos óseos aunque solo el injerto de hueso esponjoso de cresta ilíaca demostró aportar una nueva formación ósea. Hubo un mayor grado de relleno óseo en aquellos defectos tratados con injerto óseo respecto a las lesiones tratadas sin injerto. Desafortunadamente en muchos de los casos el relleno óseo se acompañaba de una reabsorción radicular y/o anquilosis dentaria.

El efecto del tratamiento de la regeneración de defectos de furca de grado II o III ha sido evaluado también en perros (Caffesse, Domínguez y cols., 1990; Pontoriero, Nyman y cols., 1992; Lindhe, Pontoriero y cols., 1995) En estos estudios se comparan los resultados obtenidos en las localizaciones tratadas con membranas (reabsorbibles y no reabsorbibles) con las localizaciones control. En ambos tipos de lesiones, la RTG obtuvo resultados mejores en cuanto a ganancia de nivel de inserción y relleno óseo del defecto respecto a la cirugía de acceso. Los autores (Caffesse, Domínguez y cols., 1990) concluyen que la aplicación de los principios de la RTG mejora la calidad de cicatrización de los defectos furcales de clase II. En las localizaciones control el epitelio fue la característica histológica predominante mientras que en las localizaciones test predominó la presencia de nuevo cemento, nuevo hueso y nuevo conectivo.

Diversos estudios (Pontoriero, Nyman y cols., 1992; Lindhe, Pontoriero y cols., 1995) demuestran que es posible obtener un completo cierre de las furcas de grado III mediante la formación de nuevo hueso y nuevo ligamento periodontal. Según los mismos, las membranas reabsorbibles eran igualmente efectivas en comparación con las membranas no reabsorbibles. Similares resultados encuentran Araujo y Lindhe (1998) donde comparan los resultados obtenidos tras el tratamiento de RTG en furcas de grado III en el mismo modelo animal y utilizando dos tipos diferentes de membranas reabsorbibles (Guidor ${ }^{\circledR}$ y Resolut ${ }^{\circledR}$ ). Los resultados demostraron que todas las superficies radiculares tratadas estaban cubiertas por cemento. En todas las muestras el cemento es de tipo celular, tanto de fibras intrínsecas como de fibras extrínsecas. En el grupo tratado con Resolut ${ }^{\circledR}$ gran parte del defecto estaba ocupado por hueso mineralizado mientras que en todas las muestras del grupo tratado con Guidor ${ }^{\circledR}$ acaeció la formación de un granuloma. Los autores concluyen que la proporción de nuevo hueso mineralizado y de nuevo ligamento periodontal es 3-4 veces superior en el grupo Resolut ${ }^{\circledR}$. El tipo de cemento presente en los dos grupos es comparable (cemento acelular de fibras extrínsecas/intrínsecas). Los mismos autores publican en el mismo año un estudio (Araujo and Lindhe 1998) donde esta vez comparan la regeneración periodontal obtenida con RTG (Control) versus RTG junto con EMD (Test) en tratamiento de furcas de grado III. Los resultados llevan a concluir que la calidad y la cantidad de tejido formado de novo resultan muy similares entre test y control. La diferencia substancial reside en que en el grupo control se forma solo cemento celular de fibras extrínsecas/intrínsecas y no hay diferencia entre la porción coronal/apical de la furcación. En el grupo test se forma cemento acelular de fibras extrínsecas/intrínsecas en la porción más apical. En ambos grupos el ligamento periodontal de la zona apical presenta más colágeno, menos estructuras vasculares y tejido residual. Donos y cols., en 2003 utilizan monos ("Macaca Fascicularis") para comparar los resultados de la regeneración periodontal con RTG versus EMD versus RTG más EMD en el tratamiento de furcas grado III.

En relación a los resultados obtenidos cabe destacar que el tratamiento con EMD aporta una tasa de regeneración no predecible en cuanto a ganancia de nueva inserción mientras que se obtiene un buen porcentaje de relleno óseo. Los resultados con el tratamiento combinado de RTG más EMD son muy parecidos a los obtenidos con RTG solo, demostrando un escaso efecto añadido del EMD junto con las membranas.

\section{¿EXISTE EVIDENCIA DE REGENERACIÓN EN MODELOS CLÍNICOS?}

Para una mejor interpretación de los resultados del análisis de los estudios clínicos que versan sobre re- 
generación periodontal aplicada en molares con afectación furcal, estratificaremos los estudios según el tipo de furcación (grado II y III) y según se haya procedido en mandíbula o en maxilar.

\section{Grado II}

\section{Mandibular}

El tratamiento de lesiones de furcación mediante el uso de EMD ha sido estudiado principalmente en furcas de grado II (Fig. 2). Donos y cols., 2003 (Donos cols., 2003). Este grupo utiliza una serie de casos tratados con EMD con reevaluación de los defectos a 36 meses. El tratamiento de los defectos tanto bucales como linguales no conllevó en ningún caso a un cierre completo de las furcas. La ganancia del nivel de inserción fue ligeramente mayor en bucal respecto a lingual aunque los resultados clínicos fueron poco satisfactorios. Un estudio recientemente publicado que compara los resultados obtenidos con EMD versus aquellos obtenidos con cirugía de acceso, (Chitsazi y cols., 2007) demuestra que la asociación de EMD con el colgajo de acceso aporta una mejora en cuanto al parámetro de ganancia de inserción horizontal respecto al control. Una serie de artículos recientemente publicados demuestra que la aplicación de $\mathrm{EMD}^{\circledR}$ resulta en una reducción estadísticamente significativa superior del componente óseo horizontal de la furcación respecto a RTG (Resolut ${ }^{\circledR}$ ). EMD ${ }^{\circledR}$ cicatriza con una menor recesión de tejido blando respecto a RTG (Resolut ${ }^{\circledR}$ ). Evaluando los factores relacionados con el paciente, encuentran que, en pacientes de mayor edad ( $>54$ años), varones, fumadores, y con peor control de pla$\mathrm{ca}, \mathrm{EMD}^{\circledR}$ obtiene mejores resultados en cuanto a las variables estudiadas respecto a RTG (Resolut ${ }^{\circledR}$ ) (Jepsen y cols., 2004; Meyle y cols., 2004; Hoffmann y cols., 2006).

Los estudios que han evaluado el potencial de la regeneración en este tipo de lesión mediante el uso de membranas aportan resultados dispares. Los mejores resultados disponibles en la literatura son aquellos obtenidos por el grupo de investigadores suecos (Pontoriero y cols., 1988). En este estudio comparan los resultados obtenido con RTG versus el colgajo de acceso. Para todos los parámetros medidos: "ganancia de inserción vertical, ganancia de inserción horizontal y reducción de profundidad de sondaje", el empleo de las membranas (Gore -Tex ${ }^{\circledR}$ ) proporcionaba mejores resultados. Además en el $67 \%$ de los casos tratados con RTG obtenían un cierre completo de la lesión. Aun- que otros estudios refieran buenos resultados en cuanto a los parámetros clínicos arriba mencionado, en ninguno de ellos se han obtenido porcentajes tan elevados de cierre completo de las furcaciones (Lekovicy cols., 1989; Paul, Mellonig y cols., 1992; Caton y cols., 1994; Wang y cols., 1994).

Otros ensayos clínicos han comparado el potencial regenerativo de las membranas versus las membranas junto con injertos óseos. En estos casos la colocación del injerto óseo mejoraba ligeramente los parámetros clínicos de ganancia de inserción y ósea vertical mientras que no se encuentran diferencias estadísticamente significativas entre los dos tratamientos para ganancia de inserción y de hueso medido de forma horizontal. Destacando que en ningún caso obtienen un cierre completo de las furcas (Lekovic, Kenney y cols., 1990; Anderegg, Martin y cols., 1991; Wallace, Gellin y cols., 1994).

\section{Maxilar}

De nuevo los mejores resultados publicados sobre el tratamiento regenerativo en furcaciones de grado II maxilares (Fig. 2)son aquellos publicado por Pontoriero y Lindhe en 1995. En este ensayo clínico aleatorizados aquellas furcas tratadas con una membrana de Gore$\mathrm{Tex}^{\circledR}$ resultaban en una mejora estadísticamente significativa un cuanto a ganancia del nivel de inserción y al relleno óseo demostrado con reentrada quirúrgica. Estos resultados fueron estadísticamente significativo solamente para las furcas vestibulares y no para las furcas proximales al igual que para las furcas mandibulares otros estudios demuestran una mejora de los

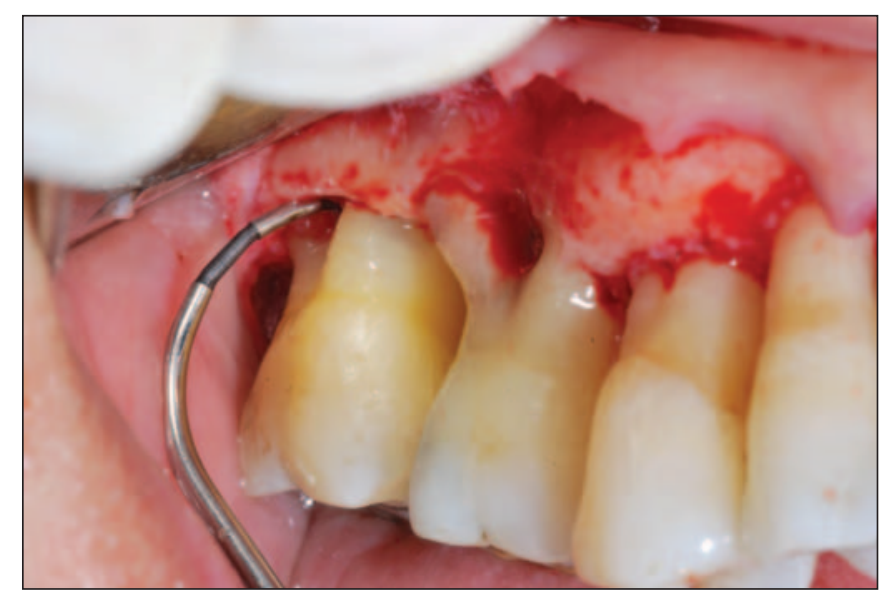

Fig. 2: Furca de grado II. 
parámetros clínico tras la utilización de las membranas (Metzler y cols., 1991; Mellonig y cols., 1994). Los resultados analizados no permiten concluir que exista un verdadero beneficio clínico en la utilización de membranas en defectos de grado II maxilar.

\section{Grado III}

\section{Mandibular y maxilar}

Debido a la limitada información científica sobre el tratamiento de este tipo de lesiones furcales, nos limitaremos a describir los dos ensayos clínicos aleatorizados que refieren los mejores resultados disponibles en la literatura (Pontoriero y cols., 1989; Pontoriero y Lindhe 1995).

$\mathrm{Al}$ igual que en otros, en estos estudio citados, se trataron los pacientes con un diseño a boca partida, lesiones de furcación bilaterales. Se efectuarán las mediciones clínicas en basal del índice de placa, índice gingival, profundidad de sondaje, nivel de inserción horizontal (Fig. 3) y vertical (en las furcas maxilares) En un lado se efectuó un colgajo de acceso para el alisado de la raíz y el desbridamiento mientras que en la lesión contralateral, tras el levantamiento del colgajo y el alisado radicular, se coloca una membrana de Gore-Tex ${ }^{\circledR}$.Tras un periodo de 6 meses bajo un estricto control de placa se efectuaba un nuevo examen clínico y la reentrada quirúrgica.

En las lesiones mandibulares se obtienen mayor ganancia del nivel de inserción horizontal estadísticamente significativa respecto a las localizaciones tratadas con colgajo de acceso. En el 38\% de las localizaciones tra-

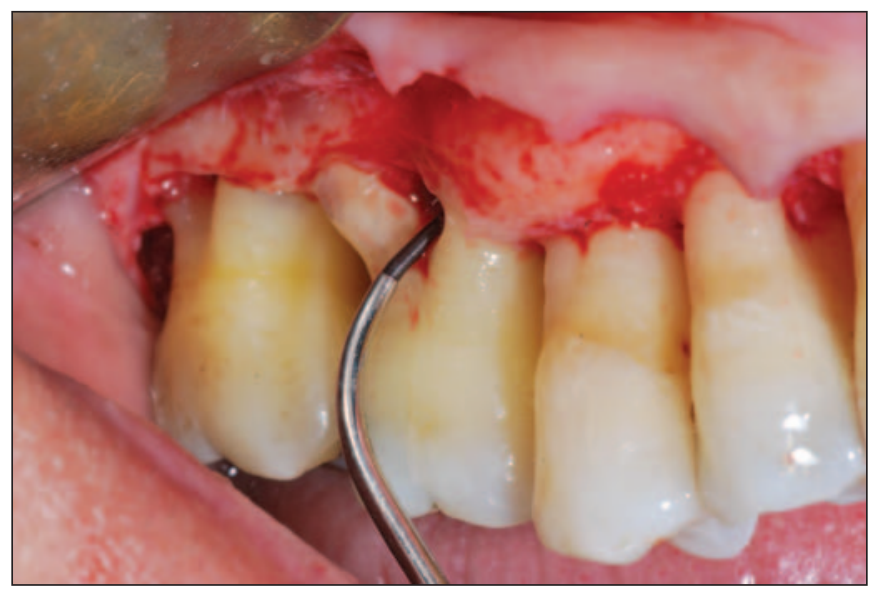

Fig. 3: Furca de grado III. tadas con RTG se obtiene un cierre completo de las furcas y en el $47 \%$ se obtiene un cierre parcial. No existen estudios que confirmen estos resultados. En cuanto a las lesiones de furcación de grado III maxilares los resultados parecen ser menos esperanzadores. En ningún caso se obtuvo un cierre completo de la lesión aunque las localizaciones tratadas con membranas resultaron en una mejora estadísticamente significativa de los parámetros clínicos.

\section{¿EXISTE PREDICTIBILIDAD EN CUANTO A LA REGENERACIÓN DE LESIONES FURCALES DE DISTINTO GRADO: I, IIY III MAXILARES Y MANDIBULARES?}

\section{Grado II mandibular}

- Mejora de los parámetros clínicos con membranas no reabsorbibles (De clase II a clase I) aunque hay variabilidad entre los estudios en cuanto a cierre completo de la lesión. Resultados parecidos se obtienen con membranas reabsorbibles.

- El uso conjunto de membranas e injertos óseo no aporta mejora en los parámetros clínicos.

\section{Grado II maxilar}

- Resultados muy poco predecibles, con alguna mejora de los parámetros clínicos en furcas vestibulares. Muy pocos defectos se resuelven por completo.

Grado III mandibular y maxilar

- Poco predecible e inconsistente respuesta al tratamiento regenerativo.

\section{DISCUSIÓN}

Gracias a la investigación científica se ha producido en la última década grandes progresos técnicos y una evolución en el diagnóstico de la enfermedad junto con un mejor entendimiento de la patogénesis de la enfermedad periodontal. Estos avances han generado un cambio en el enfoque terapéutico y una modificación en el plan de tratamiento. Pese a todo, el tratamiento de las lesiones furcales sigue representando un reto para el clínico. Analizando la literatura disponible podemos encontrar una amplia gama de tratamientos incluyendo las más recientes técnicas quirúrgicas como los procedimientos de regeneración tisular (Jepsen y cols., 2002; Jepsen y cols., 2004).

Tras la revisión de la literatura disponible, cabe destacar la heterogenicidad de los resultados obtenidos en los 
estudios analizados y entre los distintos trabajos sintetizados. Una posible explicación a esta heterogenicidad puede estar basada en la variabilidad entre estudios en cuanto a los factores documentados que han demostrado influir en el resultado de la cirugía regenerativa (Machtei y Schallhorn 1995; Sanz y Giovannoli, 2000), entre los cuales cabe destacar: la higiene oral, el hábito tabáquico y la morfología del defecto. También podrían estar influenciados estos resultados por la técnica quirúrgica aplicada y la elección del material de regeneración en combinación o no con una material de barrera. Además parece factible que los cuidados posquirúrgicos en cuanto al control de placa, el control de la infección y el uso de profilaxis antibiótica sistémica pueda tener un efecto significativo en los resultados obtenidos. Por último destacar la variabilidad entre estudios en cuanto a las mediciones clínicas y radiográficas realizadas en relación a las referencias utilizadas para medir la altura, profundidad y anchura de los defectos.

El compendio de todos estos factores lleva a evidenciar la necesidad del desarrollo de una mayor investigación de los factores pronósticos para poder explicar la heterogenicidad.

Dado que ningún tratamiento demuestra de forma fehaciente ser superior a los demás en términos absolutos de supervivencia dentaria, no se debería sustraer conclusiones o protocolos de decisión basados en esta revisión. Pese a esto basándonos en los datos obtenidos se pueden establecer ciertas directrices generales con respecto al tratamiento de las lesiones de furca en molares.

El tratamiento conservador no quirúrgico ha demostrado ser efectivo en furcas de grado I para evitar la progresión de la pérdida de soporte. En caso de progresión, con un incremento de la pérdida de inserción esta terapia presenta ciertas limitaciones entre las cuales destaca la inefectividad en la eliminación del cálculo en la furca (Matia y cols., 1986; Fleischer y cols., 1989) En relación a esto, los pacientes encuentran grandes dificultades en la higiene de las furcas de grado II y III, de este modo el tratamiento no quirúrgico de estas localizaciones es en la mayoría de los casos inefectivo, conduciendo en general a la pérdida dentaria (Dannewitz y cols., 2006; Carnevale y cols., 2007). Siendo necesario en la mayoría de los casos un cambio en la anatomía interradicular para el correcto acceso y mantenimiento de estas localizaciones.

Pasando a analizar los factores pronósticos que afectan al resultado de la terapia cabría destacar la severi- dad inicial de la lesión furcal (Pretzl, Kaltschmitt y cols., 2008), además de la amplitud de los periodos de tiempo entre visita y visita durante la terapia periodontal de mantenimiento (Rosen y cols., 1999).

El mal control de placa y el consumo de tabaco no solo se relacionan con la progresión de la enfermedad periodontal (Heitz-Mayfield 2005) sino que además están asociados con un incremento en la prevalencia de lesiones furcales (Mullally y Linden 1996). La susceptibilidad individual frente a la enfermedad periodontal también podría estar influenciada por factores genéticos (Michalowicz y cols., 1991) aunque no se haya establecido una relación directa entre la los factores genéticos y una diferente supervivencia de dientes asociados a lesiones furcales.

Los motivos más frecuentes de pérdida dentaria están en su mayoría relacionados con fracturas radiculares, complicaciones endodónticas y lesiones cariosas en la región furcal, y no con la recurrencia de la enfermedad periodontal.

En relación a la terapia regenerativa con los principios de la regeneración tisular guiada, los distintos autores citados obtienen un ratio de supervivencia de entre un $83,3 \%$ a un $100 \%$ en el tratamiento de defectos furcales (Jepsen y cols., 2002; Jepsen y cols., 2004). Es posible obtener mediante RTG mejoras en cuanto al sondaje horizontal en furcas de grado II mandibulares a diferencia de los resultados en furcas maxilares donde no se obtienen mejoría o mínimas y poco predecibles. En referencia a las furcas de grado III hasta la actualidad no hay datos para poder afirmar la predictibilidad del tratamiento de RTG para el cierre de la furca.

Una alternativa al tratamiento de RTG dentro de la terapia regenerativa de molares con lesiones furcales es el uso de proteínas de la matriz derivadas del esmalte. Hasta la actualidad solo unos pocos estudios han abordado el tratamiento de las furcas con esta técnica. Obteniendo en la mayoría de los casos una reducción horizontal de la furca siendo poco predecible el cierre completo de ésta.

\section{CONCLUSIONES}

\section{Implicaciones prácticas}

- La colocación de una membrana para la regeneración tisular guiada puede mejorar la condición clí- 
nica de las furcas maxilares y mandibulares de Clase II.

- Las mejoras obtenidas con la cirugía de acceso son limitadas o inexistentes para el tratamiento de furcas de grado II maxilares y mandibulares.

- La RTG obtiene resultados estadísticamente superiores a los obtenidos con el colgajo de acceso y equiparables al EMD en relación al cierre horizontal y vertical de la furca.

- Existe limitada información respecto a la regeneración en furcas de grado III con unos resultados con una muy baja predictibilidad.

- El cierre completo de la lesión furcal no se consigue de forma predecible ni con RTG ni con EMD en molares maxilares ni mandibulares.

- Las diferencias obtenidas en los resultados de los distintos estudios para el tratamiento de defectos de furca son debidas al control de placa por parte del paciente, al programa de mantenimiento, a la selección de los defectos y al manejo quirúrgico entre otras.

- Según la literatura revisada es posible hipotetizar que el consumo de tabaco, la administración de antibióticos y los tipos de membranas puedan tener alguna influencia sobre los resultados del tratamiento.

\section{BIBLIOGRAFÍA}

1. AAP. Glossary of periodontal terms, 3rd edition. Chicago Illinois. 1992.

2. Anderegg CR, Martin SJ y cols. Clinical evaluation of the use of decalcified freeze-dried bone allograft with guided tissue regeneration in the treatment of molar furcation invasions. J Periodontol 1991;62(4):264-8.

3. Araujo MG, Berglundh T y cols. GTR treatment of degree III furcation defects with 2 different resorbable barriers. An experimental study in dogs." J Clin Periodontol 1998;25(3):253-9.

4. Araujo M G, Lindhe J. GTR treatment of degree III furcation defects following application of enamel matrix proteins. An experimental study in dogs. J Clin Periodontol 1998; 25(6):524-30.

5. Axelsson P, Nystrom B, cols. The long-term effect of a plaque control program on tooth mortality, caries and periodontal disease in adults. Results after 30 years of maintenance. J Clin Periodontol 2004;31(9):74957.
6. Bissada NF, Abdelmalek RG. Incidence of cervical enamel projections and its relationship to furcation involvement in Egyptian skulls. J Periodontol 1973;44 (9):583-5.

7. Caffesse RG, Dominguez LE y cols. Furcation defects in dogs treated by guided tissue regeneration (GTR). J Periodontol 1990:61(1): 45-50.

8. Carnevale G, Cairo F y cols. Long-term effects of supportive therapy in periodontal patients treated with fibre retention osseous resective surgery. I: recurrence of pockets, bleeding on probing and tooth loss. J Clin Periodontol 2007;34(4): 334-41.

9. Carnevale G, Pontoriero R y cols. Long-term effects of root-resective therapy in furcation-involved molars. A 10-year longitudinal study. J Clin Periodontol 1998;25(3):209-14.

10. Caton J, Greenstein G y cols. Synthetic bioabsorbable barrier for regeneration in human periodontal defects. J Periodontol 1994;65(11):1037-45.

11. Caton JG, Greenstein G. Factors related to periodontal regeneration. Periodontol 1993;2000(1):9-15.

12. Chitsazi, M.T., R. Mostofi Zadeh Farahani, y cols. Efficacy of open flap debridement with and without enamel matrix derivatives in the treatment of mandibular degree II furcation involvement. Clin Oral Investig 2007; 11(4):385-9.

13. Dannewitz B, Krieger JK y cols. Loss of molars in periodontally treated patients: a retrospective analysis five years or more after active periodontal treatment. J Clin Periodontol 2006;33(1):53-61.

14. Donos N, Glavind L y cols. Clinical evaluation of an enamel matrix derivative in the treatment of mandibular degree II furcation involvement: a 36-month case series. Int J Periodontics Restorative Dent 2003;23(5): 507-12.

15. Donos N, Sculean A y cols. Wound healing of degree III furcation involvements following guided tissue regeneration and/or Emdogain. A histologic study.J Clin Periodontol 2003;30(12):1061-8.

16. Eickholz P, Hausmann E. Evidence for healing of periodontal defects 5 years after conventional and regenerative therapy: digital subtraction and bone level measurements. J Clin Periodontol 2002;29(10):922-8. 
17. Eickholz P, Pretzl B y cols. Long-term results of guided tissue regeneration therapy with non-resorbable and bioabsorbable barriers. III. Class II furcations after 10 years. J Periodontol 2006;77(1):88-94.

18. Ellegaard B, Karring T y cols. New attachment after treatment of interradicular lesions. J Periodontol 1973; 44(4):209-17.

19. Ellegaard B, Karring T y cols. The fate of vital and devitalized bone grafts in the healing of interradicular lesions. J Periodontal 1975;Res 10(2):88-97.

20. Fleischer HC, Mellonig JT y cols. Scaling and root planing efficacy in multirooted teeth.J Periodontol 1989; 60(7):402-9.

21. Hamp SE, Nyman S y cols. Periodontal treatment of multirooted teeth. Results after 5 years. J Clin Periodontol 1975;2(3):126-35.

22. Heitz-Mayfield LJ. Disease progression: identification of high-risk groups and individuals for periodontitis. J Clin Periodontol 2005;32 Suppl 6: 196-209.

23. Hoffmann T, Richter S y cols. A randomized clinical multicentre trial comparing enamel matrix derivative and membrane treatment of buccal class II furcation involvement in mandibular molars. Part III: patient factors and treatment outcome. J Clin Periodontol 2006:33(8): 575-83.

24. Huynh-Ba G, Kuonen P y cols. The effect of periodontal therapy on the survival rate and incidence of complications of multirooted teeth with furcation involvement after an observation period of at least 5 years: a systematic review. J Clin Periodontol 2009;36(2):164-76.

25. Jepsen S, Eberhard J y cols. A systematic review of guided tissue regeneration for periodontal furcation defects. What is the effect of guided tissue regeneration compared with surgical debridement in the treatment of furcation defects? J Clin Periodontol 2002;29 Suppl 3:103-16; discussion 160-2.

26. Jepsen S, Heinz B y cols. A randomized clinical trial comparing enamel matrix derivative and membrane treatment of buccal Class II furcation involvement in mandibular molars. Part I: Study design and results for primary outcomes. J Periodontol 2004;75(8):1 150-60.

27. Lang NP, Cumming BR y cols. Toothbrushing frequency as it relates to plaque development and gingival health. J Periodontol 1973;44(7):396-405.
28. Lekovic V, Kenney EB y cols. Treatment of class II furcation defects using porous hydroxylapatite in conjunction with a polytetrafluoroethylene membrane. J Periodontol 1990;61(9):575-8.

29. Lekovic V, Kenney EB y cols. Evaluation of guided tissue regeneration in Class II furcation defects. A clinical reentry study. J Periodontol 1989;60(12):694-8.

30. Lindhe J, Pontoriero R y cols. The effect of flap management and bioresorbable occlusive devices in GTR treatment of degree III furcation defects. An experimental study in dogs. J Clin Periodontol 1995;22(4): 276-83.

31. Machtei EE, Schallhorn RG. Successful regeneration of mandibular Class II furcation defects: an evidencebased treatment approach. Int J Periodontics Restorative Dent 1995;15(2):146-67.

32. Matia J. I, Bissada NF y cols. Efficiency of scaling of the molar furcation area with and without surgical access. Int J Periodontics Restorative Dent 1986;6(6):24-35.

33. McFall WT, Jr. Tooth loss in 100 treated patients with periodontal disease. A long-term study. J Periodontol 1982;53(9):539-49.

34. Mellonig JT, Seamons BC y cols. Clinical evaluation of guided tissue regeneration in the treatment of grade II molar furcation invasions. Int J Periodontics Restorative Dent 1994;14(3):254-71.

35. Metzler DG, Seamons BC y cols. Clinical evaluation of guided tissue regeneration in the treatment of maxillary class II molar furcation invasions. J Periodontol 1991;62 (6):353-60.

36. Meyle J, Gonzales JR y cols. A randomized clinical trial comparing enamel matrix derivative and membrane treatment of buccal class II furcation involvement in mandibular molars. Part II: secondary outcomes.J Periodontol 2004;75(9):1 188-95.

37. Michalowicz BS, Aeppli DP y cols. A twin study of genetic variation in proportional radiographic alveolar bone height. J Dent Res 1991;70(11):1431-5.

38. Mullally BH, Linden GJ. Molar furcation involvement associated with cigarette smoking in periodontal referrals. J Clin Periodontol 199623(7):658-61.

39. Nyman S, Lindhe J y cols. New attachment following surgical treatment of human periodontal disease. J Clin Periodontol 1982;9(4): 290-6. 
40. Paul BF, Mellonig JT y cols. Use of a collagen barrier to enhance healing in human periodontal furcation defects. Int J Periodontics Restorative Dent 1992;12 (2):123-31.

41. Pontoriero R, Lindhe J. Guided tissue regeneration in the treatment of degree II furcations in maxillary molars. J Clin Periodontol 1995;22(10):756-63.

42. Pontoriero R. Lindhe J. Guided tissue regeneration in the treatment of degree III furcation defects in maxillary molars. J Clin Periodontol 1995;22(10):810-2.

43. Pontoriero R. Lindhe J y cols. Guided tissue regeneration in degree II furcation-involved mandibular molars. A clinical study. J Clin Periodontol 1988;15(4):247-54.

44. Pontoriero, R. Lindhe J y cols. Guided tissue regeneration in the treatment of furcation defects in mandibular molars. A clinical study of degree III involvements. J Clin Periodontol 1989;16(3):170-4.

45. Pontoriero, R, Nyman S y cols. Guided tissue regeneration in surgically-produced furcation defects. An experimental study in the beagle dog." J Clin Periodontol 1992;19(3): 159-63.

46. Pretzl B, Kaltschmitt J y cols. Tooth loss after active periodontal therapy. 2: tooth-related factors. J Clin Periodontol 2008;35(2):175-82.

47. Rosen B, Olavi G y cols. Effect of different frequencies of preventive maintenance treatment on periodontal conditions. 5-Year observations in general dentistry patients. J Clin Periodontol 1999;26(4): 225-33.

48. Ross IF, Thompson RH, Jr. Furcation involvement in maxillary and mandibular molars. J Periodontol 1980; $51(8): 450-4$.
49. Sanz M, Giovannoli JL. Focus on furcation defects: guided tissue regeneration. Periodontol 2000. 2000;22: 169-89.

50. Sculean A, Nikolidakis D y cols. Regeneration of periodontal tissues: combinations of barrier membranes and grafting materials - biological foundation and preclinical evidence: a systematic review.J Clin Periodontol 2008;35 (8 Suppl):106-16.

51. Socransky, S. S. and A. D. Haffajee. Periodontal microbial ecology. Periodontol 2000. 2005;38:135-87.

52. Svardstrom G, Wennstrom JL. Periodontal treatment decisions for molars: an analysis of influencing factors and long-term outcome.J Periodontol 2000;71(4):579-85.

53. Wallace SC, Gellin RG y cols. Guided tissue regeneration with and without decalcified freeze-dried bone in mandibular Class II furcation invasions. J Periodontol 1994; 65(3):244-54.

54. Wang HL, O'Neal RB y cols. Evaluation of an absorbable collagen membrane in treating Class II furcation defects. J Periodontol 1994;65(11):1029-36.

55. Yukna RA, Yukna CN. Six-year clinical evaluation of HTR synthetic bone grafts in human grade II molar furcations. J Periodontal Res 1997;32(8):627-33.

\section{CORRESPONDENCIA}

\section{S. Fabrizi}

Federico Carlos Sainz de Robles, 15 3K 28035 Madrid, España

Teléfono: (0034) 696872110 - 913769073

Correo electrónico: simonefabrizi@gmail.com 